\title{
DETERMINAÇÃO SELETIVA DE TRIBUTILESTANHO NA PRESENÇA DE Sn(IV) EM AMOSTRAS AMBIENTAIS USANDO HG-ICP OES E Saccharomyces cerevisiae COMO MATERIAL SORVENTE
}

\author{
Monizze Vannuci e Silva, Amauri Antonio Menegario*, Mariana Franchi e Paulo Sergio Tonello \\ Centro de Estudos Ambientais, Universidade Estadual Paulista, Av. 24-A, 1515, 13506-900 Rio Claro - SP, Brasil \\ Ana Luiza Brossi-Garcia \\ Departamento de Zoologia, Instituto de Biociências, Universidade Estadual Paulista, Av. 24-A, 1515, 13506-900 Rio Claro - SP / \\ Centro de Estudos Ambientais, Universidade Estadual Paulista, Av. 24-A, 1515, 13506-900 Rio Claro - SP, Brasil
}

Recebido em 9/11/09; aceito em 15/3/10; publicado na web em 20/7/10

\begin{abstract}
SELECTIVE DETERMINATION OF TRIBUTYLTIN IN THE PRESENCE OF Sn(IV) IN ENVIRONMENTAL SAMPLES USING HGICP OES AND Saccharomyces cerevisiae AS SORBING MATERIAL. A method for selective determination of tributyltin in the presence of $\mathrm{Sn}(\mathrm{IV})$ by combining hydride generation inductively coupled plasma optical emission spectrometry (HG-ICP OES) and solid phase extraction (SPE) using baker's yeast is proposed. The procedure is based on selective retention of TBT by the yeast at $\mathrm{pH}=6$. Detection limit of $1.9 \mu \mathrm{g} \mathrm{L} \mathrm{L}^{-1}$ and quantification limit of $6.3 \mu \mathrm{g} \mathrm{L} \mathrm{L}^{-1}$ were obtained. TBT and Sn(IV) were determined in the range of $0-25 \mu \mathrm{g} \mathrm{L}^{-1}$ and the proposed approach was applied to analyze river water, sea water and biological extracts, with recoveries of 114, 101 and $86 \%$, respectively.
\end{abstract}

Keywords: SPE; TBT; HG-ICP OES.

\section{INTRODUÇÃO}

Em água do mar, o Sn pode ocorrer nas formas inorgânicas livres nos estados de oxidação $\mathrm{Sn}(\mathrm{II})$ ou $\mathrm{Sn}(\mathrm{IV})$, ou nas formas organometálicas como o TBT (tributilestanho) ou seus produtos de degradação DBT (dibutilestanho) e MBT (monobutilestanho). ${ }^{1}$ Os compostos organometálicos de estanho são mais tóxicos que as espécies inorgânicas e a toxicidade aumenta para compostos com maior número de grupos alquilas ligados ao estanho. ${ }^{2} \mathrm{~A}$ atividade biológica e toxicidade de estanho também estão associadas ao estado de oxidação das formas inorgânicas: $\mathrm{Sn}(\mathrm{II})$ é mais tóxico que $\mathrm{Sn}(\mathrm{IV}){ }^{3}$

Os compostos orgânicos de Sn entram no ambiente por meio de produtos feitos pelo homem (biocidas e estabilizantes) ou são formados a partir das espécies inorgânicas. Os compostos antropogênicos mais bem conhecidos incluem espécies de TBT, utilizadas em fórmulas de pinturas antifuligem que são responsáveis por efeitos deletérios em vários organismos marinhos, principalmente, bivalves e crustáceos. ${ }^{1}$

Pesquisas revelam que o siri azul (Callinectes sapidus) é capaz de metabolizar efetivamente o TBT, dependendo do nível encontrado no ambiente. Porém, outras espécies, como Ilyanassa obsoleta, podem desenvolver características masculinas em fêmeas, fenômeno denominado imposexo, quando a concentração de TBT no ambiente é de aproximadamente $2 \mathrm{ng} \mathrm{L}^{-1}{ }^{4}$

A cromatografia gasosa (GC) é efetiva para a separação de espécies orgânicas de estanho e possibilita limites de detecção na faixa de ng $\mathrm{L}^{-1}$ a $\mu \mathrm{g} \mathrm{L}^{-1}$ dependendo do tipo de detector utilizado. ${ }^{5-8} \mathrm{Um}$ dos principais inconvenientes desta técnica é o pré-tratamento tedioso da amostra, que envolve várias etapas de extração do analito, geralmente usando extrações líquido-líquido (LPE) e, posteriormente, a derivação do analito com tetraetilborato de sódio. ${ }^{9}$

A cromatografia líquida (LC) também é efetiva para o fracionamento de estanho orgânico e inorgânico, ${ }^{10-14}$ porém, os limites de detecção são pobres quando detectores convencionais são utilizados. O limite de detecção pode ser consideravelmente melhorado quando a LC é acoplada ao ICP-MS. ${ }^{13-15}$ No entanto, métodos envolvendo a

*e-mail: amenega@rc.unesp.br
LC requerem, pelo menos, uma etapa de pré-tratamento usando LPE antes da separação cromatográfica, mesmo para amostras de água. ${ }^{14}$

Poucos métodos não cromatográficos foram utilizados para especiação de $\mathrm{Sn}$, sendo que alguns foram baseados na extração em fase sólida, ${ }^{16-18}$ extração seletiva, ${ }^{19}$ XANES ( $X$-ray absorption nearedge structure spectroscopy), ${ }^{20}$ extração em fases líquida-líquida ${ }^{21,22}$ e diluição isotópica sub-estequiométrica. ${ }^{23}$

Bermejo-Barrera et al. ${ }^{16}$ propuseram a determinação de TBT em águas mineral e de torneira utilizando, para a retenção do TBT, a resina Amberlite XAD-2 impregnada com tropolone e posterior determinação por espectrometria de absorção atômica com atomização eletrotérmica (ET AAS). O mesmo material (resina Amberlite XAD-2 impregnada com tropolone) foi proposto para determinar $\mathrm{Sn}$ total orgânico em amostras de água marinha. ${ }^{17} \mathrm{O}$ processo também permitiu a distinção entre compostos orgânicos de estanho e Sn(IV), pois a forma inorgânica não é retida pela coluna. Os autores relataram resultados com limite de detecção de $13 \mathrm{ng} \mathrm{L}^{-1}$, desvio padrão menor que $10 \%$ e recuperação do analito próxima a $100 \%$.

Vandenberg e Khan ${ }^{18}$ propuseram um método capaz de determinar seletivamente espécies orgânicas e inorgânicas de estanho. As espécies orgânicas de $\mathrm{Sn}$ foram separadas passando-se a amostra acidificada em uma coluna C18 que adsorve todos os compostos orgânicos, deixando o Sn inorgânico em solução. O limite de detecção relatado para determinação de estanho orgânico total é de aproximadamente $10 \mathrm{pmol} \mathrm{dm}^{-3}$ (com tempo de deposição de $10 \mathrm{~min}$ ).

O uso de substratos biológicos (micro-organismos) para discriminar espécies metálicas é outra alternativa simples para conduzir análises de especiação e fracionamentos químicos. Trabalhos recentes evidenciam que estes substratos são um instrumento poderoso para amostragem específica e pré-concentração de várias espécies químicas. Dentre os vários micro-organismos utilizados para este propósito, a Saccharomyces cerevisiae pode ser destacada, em parte, devido à facilidade com que pode ser obtida em grandes quantidades e na forma desidratada e, principalmente, por ser um organismo não patogênico. $\mathrm{O}$ micro-organismo foi utilizado com sucesso na determinação seletiva de espécies de As, Cr, $\mathrm{Hg}$, Sb, Se e Cd. ${ }^{24-31}$ Nestes métodos, o As(III), Cr(III), metilmercúrio, $\mathrm{Sb}$ (III), Se(IV) e Cd(II) na presença de As(V), Cr(VI), Hg(II), Sb(V), $\mathrm{Se}(\mathrm{VI})$ e Cd-metalotioneina, respectivamente, foram quantitativamente 
(e seletivamente) retidos pela levedura. O micro-organismo também foi utilizado para determinação de $\mathrm{Sn}(\mathrm{II})$ e $\mathrm{Sn}(\mathrm{IV}) .{ }^{32}$ Neste método, foram avaliados diversos fatores que afetam a retenção dos analitos pela levedura, tais como $\mathrm{pH}$, quantidade de biomassa, temperatura e tempo de contato. $\mathrm{Em} \mathrm{pH}=2, \mathrm{o} \mathrm{Sn}(\mathrm{IV})$ foi quantitativamente retido pela levedura, enquanto o $\mathrm{Sn}$ (II) permanecia na solução. Depois da separação, o $\mathrm{Sn}$ (IV) presente na fase sólida foi determinado por espectrometria de emissão óptica com plasma indutivamente acoplado (ICP OES) introduzindo uma suspensão do material diretamente no sistema de nebulização do equipamento. Os limites de detecção do estanho nas fases sólida e líquida foram, respectivamente, 1,1 e $0,7 \mu \mathrm{g} \mathrm{L}^{-1}$. O método foi utilizado com sucesso para a determinação de $\mathrm{Sn}(\mathrm{II})$ e $\mathrm{Sn}$ (IV) em águas fluviais e amostras de águas residuais de indústrias (com e sem tratamento).

O uso da técnica de geração de hidretos (HG) para substituir o sistema de introdução de amostras convencional da ICP OES (nebulização pneumática) apresenta algumas vantagens, como o aumento significativo da sensibilidade (de 20 a 50 vezes) e a separação dos analitos da matriz potencializando, assim, análises mais sensíveis e seletivas. Algumas das aplicações recentes do acoplamento da HG com o ICP (HG-ICP OES) envolveram determinação de $\mathrm{Sb}\left(\mathrm{Sb}^{3+} \mathrm{e} \mathrm{Sb}^{5+}\right)$ em amostras farmacêuticas, ${ }^{33}$ de água e efluentes; ${ }^{34}$ determinação de Se em material biológico, ${ }^{35}$ determinação de As e Se em sedimentos estuarinos, ${ }^{36}$ determinações multielementares (As, $\mathrm{Pb}, \mathrm{Sb}$ e $\mathrm{Se}$ ) usando sistemas tipo "dual", ${ }^{37,38}$ que combinam a geração de hidretos e a nebulização pneumática ${ }^{37}$ ou ultrassônica ${ }^{38}$ para elementos que não geram hidretos. Para determinação de Sn, a HG-ICP OES foi utilizada com sucesso para a análise de manganês eletrolítico, de material vegetal e solos. ${ }^{39-41}$

Este trabalho teve como principal objetivo a avaliação da capacidade da Saccharomyces cerevisiae em segregar TBT na presença de Sn inorgânico e o desenvolvimento de um método analítico para determinação de TBT na presença de Sn(IV) em amostras de interesse ambiental, utilizando esse micro-organismo em associação a um sistema de HG-ICP OES.

\section{PARTE EXPERIMENTAL}

\section{Equipamentos e acessórios}

Foi utilizado um espectrômetro de emissão óptica com plasma acoplado indutivamente, marca GBC modelo integra XL (Melbourne, Austrália), equipado com uma câmara de nebulização do tipo ciclônica (Glass Expansion, Melbourne, Austrália) e nebulizador tipo V-Groove (Glass Expansion). Alternativamente, foi utilizado sistema de geração de hidretos. O sistema de geração de hidretos (fluxo contínuo) foi montado com uma bomba peristáltica, câmara de separação de fases líquida-gasosa (semelhante ao descrito por Menegário e Giné $)$, tubos de polietileno e tubos de bombeamento de Tygon. Neste sistema, a amostra acidificada confluiu diretamente com a solução de $\mathrm{NaBH}_{4}$ Os gases gerados, depois de separados da fase líquida foram conduzidos ao ICP com um fluxo de argônio. Os parâmetros instrumentais do ICP OES e as condições de geração de hidretos são apresentados na Tabela 1.

Outros equipamentos e acessórios utilizados foram: centrífuga Jouan, modelo B4i (St. Herblain, França), banho térmico ITR, modelo 80 (Esteio, Brasil), bloco aquecedor Tecnal, modelo TE - 40/25 (Piracicaba, Brasil), banho de ultrassom Bandelin, modelo Sonorex RK 102, com frequência de $35 \mathrm{kHz}$ (Berlim, Alemanha) e evaporador rotatório a vácuo Quimis, modelo Q.344.2 (São Paulo, Brasil).

\section{Reagentes e soluções}

Em todo o trabalho foram utilizados reagentes de grau analítico e água purificada em um sistema deionizador Milli-Q (Millipore, Bedford, MA, EUA) com resistividade de $18.2 \mathrm{M} \Omega \mathrm{cm}$.
Tabela 1. Condições de operação do ICP-OES, nebulização pneumática e geração de $\mathrm{SnH}_{4}$

\begin{tabular}{lcc}
\hline ICP OES & & \\
\hline Potência, $\mathrm{W}$ & $\begin{array}{c}\text { incidente } \\
\text { plasma } \\
\text { Vazão dos gases, } \mathrm{L} \mathrm{min}{ }^{-1}\end{array}$ & 1200 \\
& auxiliar & 0,5 \\
Altura de observação, mm & 10 \\
Vista & radial & \\
Linha de emissão, nm & $\mathrm{Sn}$ & 189,926 \\
Nebulização Pneumática & & \\
Vazão do gás de nebulização, $\mathrm{L} \mathrm{min}^{-1}$ & & 0,6 \\
Vazão de introdução da amostra, $\mathrm{mL} \mathrm{min}^{-1}$ & & 2,8 \\
Geração de Hidretos & & \\
Vazão de NaBH & \\
Vazão da amostra, $\mathrm{mL} \mathrm{min}^{-1}$ & & 1,4 \\
Vazão do gás de arraste, $\mathrm{L} \mathrm{min}^{-1}$ & & 2,6 \\
\hline
\end{tabular}

Foram preparadas soluções padrão estoque com concentração de $1000 \mathrm{mg} \mathrm{L}^{-1}$ de $\mathrm{Sn}(\mathrm{IV})$ e TBT a partir de Sn metálico (Aldrich, Milwaukee, EUA) e cloreto de TBT (Aldrich), respectivamente. As soluções padrão de trabalho foram preparadas por diluições das suas respectivas soluções padrão estoque com água. Os valores dos pHs das amostras e das soluções padrão foram ajustados utilizando-se soluções de $\mathrm{NaOH}$ ou $\mathrm{HCl}$ (Synth, São Paulo, Brasil).

Além das soluções estoque de $\mathrm{Sn}(\mathrm{IV})$ e TBT, foram utilizadas soluções padrão de estanho com concentrações entre $250-1000 \mu \mathrm{g}$ $\mathrm{L}^{-1}$, preparadas a partir de uma solução padrão multielementar de 100 mg L ${ }^{-1}$, em $\mathrm{HNO}_{3} 5 \%$, certificada pela QHEMIS High Purity. Essas soluções foram usadas para avaliar, periodicamente, as concentrações das soluções teste.

O preparo das soluções contendo $\mathrm{Cu}(\mathrm{II}), \mathrm{Fe}(\mathrm{III}), \mathrm{Cd}(\mathrm{II}), \mathrm{Mn}(\mathrm{II})$, $\mathrm{Zn}(\mathrm{II}), \mathrm{Ca}(\mathrm{II}), \mathrm{Mg}(\mathrm{II}), \mathrm{K}(\mathrm{I})$ e $\mathrm{Na}(\mathrm{I})$, utilizadas nos testes de interferência, foi feito a partir da dissolução de $\mathrm{CuSO}_{4} .5 \mathrm{H}_{2} \mathrm{O}$ (Merck, Darmstadt, Alemanha), $\mathrm{FeNH}_{4}\left(\mathrm{SO}_{4}\right)_{2} \cdot 12 \mathrm{H}_{2} \mathrm{O}$ (Synth), $\mathrm{Cd}\left(\mathrm{NO}_{3}\right)_{2}$ (High-Purity Standards, Charleston, EUA), $\mathrm{MnSO}_{4} \cdot \mathrm{H}_{2} \mathrm{O}$ (Synth), $\mathrm{ZnSO}_{4} \cdot 7 \mathrm{H}_{2} \mathrm{O}$ (Vetec, Duque de Caxias, Brasil), $\mathrm{CaCO}_{3}$ (Merck), $\mathrm{MgCl}_{2} \cdot \mathrm{H}_{2} \mathrm{O}$ (Mallinckrodt, EUA), $\mathrm{KCl}$ (Synth), e $\mathrm{NaCl}$ (Synth), respectivamente.

Soluções de $\mathrm{NaBH}_{4} 3 \%$ (m/v) (MP Biomedicals, EUA) em 0,05 mol L-1 de $\mathrm{NaOH}$ (Synth), usadas no sistema de geração de hidretos, foram preparadas imediatamente antes das análises.

Todos os testes foram realizados utilizando a levedura Saccharomyces cerevisiae desidratada, obtida no comércio (Fermix, São José dos Campos, Brasil).

\section{Procedimento geral de extração em fase sólida}

Todas as extrações em fase sólida foram realizadas em batelada, usando um mesmo lote da levedura Saccharomyces cerevisiae. O procedimento consistiu em adicionar alíquotas de $10 \mathrm{~mL}$ das amostras ou soluções teste de Sn(IV) e/ou TBT, em um tubo de centrífuga contendo uma quantidade determinada do substrato. Em seguida, os tubos foram vigorosamente agitados e mantidos em banho (de água) com controle de temperatura por um tempo estabelecido. Após o período de incubação, as suspensões foram centrifugadas por $7 \mathrm{~min}$ a $4000 \mathrm{rpm}$ para separação das fases.

\section{Procedimentos para determinação de Sn nas fases sólida e líquida}

Num teste de recuperação de Sn com nebulização direta da fase líquida, verificou-se que o Sn pôde ser determinado com um limite 
de detecção de $16 \mu \mathrm{g} \mathrm{L}^{-1}(3 \sigma)$ e uma interferência no processo de nebulização desprezível, apresentando recuperação de $107 \pm 4 \%$. Assim, nos testes iniciais (visando avaliar os efeitos dos parâmetros da extração em fase sólida) os valores das massas de Sn(IV) e TBT retidos na fase sólida $\left(m_{F S}\right)$ foram calculados a partir da diferença entre as massas das soluções teste (massa inicial, $m_{i}$ ) e as massas determinadas na fase líquida $\left(m_{F L}\right)$.

Os valores de $m_{F S}$ foram calculados a partir das concentrações de Sn na fase líquida usando a seguinte equação:

$$
\mathrm{m}_{\mathrm{FS}}=\mathrm{m}_{\mathrm{i}}-\mathrm{m}_{\mathrm{FL}}
$$

onde: $m_{F L}=$ é a massa de $\operatorname{Sn}(\mathrm{IV})$ ou TBT na fase líquida.

Os valores de $m_{F L}$ e $m_{i}$ podem ser obtidos pelas equações abaixo:

$$
\begin{aligned}
& \mathrm{m}_{\mathrm{FL}}=\mathrm{V} \cdot\left[\mathrm{Sn}_{\mathrm{det}}\right] \\
& \mathrm{m}_{\mathrm{i}}=\mathrm{V} \cdot\left[\mathrm{Sn}_{\text {inicial }}\right]
\end{aligned}
$$

onde: $\mathrm{V}=$ volume da solução teste de TBT ou $\mathrm{Sn}(\mathrm{IV})$ utilizada na extração; $\left[\mathrm{Sn}_{\text {inicial }}\right]$ = a concentração da solução teste de TBT ou $\mathrm{Sn}$ (IV) utilizada na extração; $\left[\mathrm{Sn}_{\text {det }}\right]=$ a concentração $\mathrm{Sn}$ (TBT ou $\mathrm{Sn}(\mathrm{IV})$ ) determinada na fase líquida.

Apesar de ser mais propensa à propagação de erros analíticos, a determinação indireta da massa retida pelo material sorvente, segundo o procedimento descrito acima, evita a análise da fase sólida. A determinação direta de Sn na fase sólida, mesmo podendo ser facilmente conduzida através da introdução de uma suspensão do material diretamente no sistema de nebulização do ICP, é sujeita a efeitos de matriz. ${ }^{32}$

Nos testes envolvendo a geração de hidretos, as determinações de $\mathrm{Sn}(\mathrm{IV})$ e TBT nas fases líquida e sólida foram realizadas depois de uma etapa de digestão.

As fases líquida e sólida foram transferidas para tubos de digestão, receberam a adição de ácido nítrico e foram aquecidas em bloco aquecedor. À fase líquida foram acrescidos $2 \mathrm{~mL}$ de ácido nítrico concentrado, enquanto que para a fase sólida foram adicionados 1 $\mathrm{mL}$ de ácido nítrico concentrado e $1 \mathrm{~mL}$ de água. Os tubos foram aquecidos a $140{ }^{\circ} \mathrm{C}$ por 120 min e, então, deixados esfriar à temperatura ambiente. A fase líquida foi então avolumada com água para $20 \mathrm{~mL}$ e a fase sólida para $10 \mathrm{~mL}$. Após esse processo, as amostras foram introduzidas em um sistema de fluxo para geração de hidretos.

No sistema de geração de hidretos, a solução da amostra confluiu com solução de $\mathrm{NaBH}_{4} 3 \%$ (m/v) e, após passar pela câmara de separação de fases, o hidreto gerado foi conduzido para o ICP através de um fluxo de argônio.

\section{Preparo das amostras}

\section{Amostras de água}

Foram coletadas 2 amostras de água durante o período chuvoso (dezembro de 2007 a março de 2008). Uma amostra de água doce, proveniente do rio Corumbataí (Rio Claro/SP) e a outra, de água salgada (Bertioga/SP). As amostras foram transportadas e preservadas sob refrigeração $\left(4^{\circ} \mathrm{C}\right)$. No laboratório, as amostras foram filtradas em membrana de acetato de celulose $(0,45 \mu \mathrm{m})$, o $\mathrm{pH}$ ajustado para $6,0 \pm 0,5$ e, então, submetidas aos processos de extração em fase sólida, digestão e geração de hidretos.

\section{Amostras de brânquias de siri azul}

Foram coletados 10 animais da espécie Callinectes sapidus, popularmente conhecidos como "siri azul", no canal de navegação de Santos/SP, por meio de armadilhas do tipo covo e por catação manual.
Os indivíduos foram mortos por congelamento e transportados ao laboratório sob refrigeração, onde foram dissecados e deles retiradas as brânquias, que foram maceradas.

A extração de TBT das brânquias foi realizada através de um procedimento similar ao descrito por Quevauviller, ${ }^{43} \mathrm{o}$ qual relatou recuperações de $85 \%$ para o TBT.

Em tubos de $15 \mathrm{~mL}$ foram adicionados $0,33 \mathrm{~g}$ do tecido macerado, $10 \mathrm{~mL}$ de metanol e $0,33 \mathrm{~mL}$ de $\mathrm{HCl}$ concentrado. A mistura foi homogeneizada e levada ao banho de ultrassom por 15 min. Em seguida, a amostra foi centrifugada por $10 \mathrm{~min}$ a 3000 rpm, sendo a fase sólida descartada no final do processo. A fase líquida foi submetida à evaporação (em evaporador rotatório a vácuo, $50-55^{\circ} \mathrm{C}$ ), até o volume final de aproximadamente $1 \mathrm{~mL}$. $\mathrm{O}$ resíduo foi retomado com água, o $\mathrm{pH}$ ajustado a $6,0 \pm 0,5 \mathrm{e}$ avolumado a $13 \mathrm{~mL}$.

\section{RESULTADOS E DISCUSSÃO}

\section{Efeito dos parâmetros de extração em fase sólida}

Estudos previamente realizados mostraram que em extrações conduzidas com uma cepa de Saccharomyces cerevisiae (liofilizada) derivada da linhagem ATCC-FL97G3 em pH 6 (tempo de contato de $60 \mathrm{~min} ; 25^{\circ} \mathrm{C}$ e $0,5 \mathrm{~g}$ de biomassa) a maior parte do $\mathrm{Sn}$ inorgânico, $\mathrm{Sn}(\mathrm{IV})$ e Sn (II), permanece em solução, enquanto o TBT é retido quase quantitativamente $(>90 \%)$ pelo substrato. ${ }^{32,44}$ Deste modo, os estudos visando avaliar o efeito das condições de extração (tempo de contato entre o material sorvente e os analitos, quantidade de biomassa e temperatura) foram conduzidos com soluções de TBT ou Sn(IV) preparadas em $\mathrm{pH}$ 6,0 $\pm 0,5$.

As retenções (Ret) de $\mathrm{Sn}(\mathrm{IV})$ e TBT mostradas nos itens subseqüentes foram calculadas a partir da seguinte equação:

$$
\text { Ret }=\left(\mathrm{m}_{\mathrm{FS}} / \mathrm{m}_{\mathrm{i}}\right) \times 100
$$

onde: $m_{F S}=$ a massa de Sn(IV) ou TBT na fase sólida; $m_{i}=$ a massa de $\mathrm{Sn}(\mathrm{IV})$ ou TBT contidos nas soluções teste.

\section{Efeito do tempo de contato}

Os testes de avaliação do tempo de contato da levedura com as soluções foram realizados nas seguintes condições: temperatura de incubação de $25^{\circ} \mathrm{C}$ e quantidade de biomassa equivalente a $0,125 \mathrm{~g}$.

A Figura 1 apresenta a retenção dos analitos na fase sólida em função do tempo de contato. Verificou-se que pelo menos $91,7 \%$ do TBT foram retidos pela levedura e que a recuperação do composto na fase líquida, em todas as condições testadas, foi menor que o LD $\left(16 \mu \mathrm{g} \mathrm{L}^{-1}\right)$ utilizando a nebulização direta. Para o $\mathrm{Sn}(\mathrm{IV})$, a retenção foi crescente com o aumento do tempo de contato.

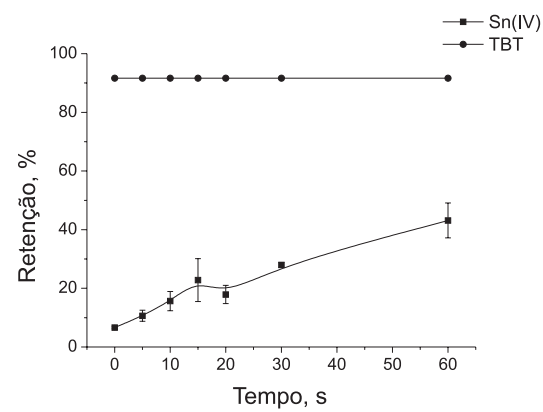

Figura 1. Efeito do tempo de contato na retenção de TBT e Sn(IV). Temperatura $=25^{\circ} \mathrm{C}$; biomassa $=0,125 \mathrm{~g} ; \mathrm{pH}=6 \pm 0,5$ 
Com base nos resultados apresentados na Figura 1 optou-se pela condução dos experimentos subsequentes utilizando extração imediata, sem tempo de contato, onde houve a retenção mínima de $\mathrm{Sn}(\mathrm{IV})$ e retenção superior a $91 \%$ do TBT pela levedura.

\section{Efeito da quantidade de biomassa}

Para avaliar o efeito da quantidade do material sorvente na separação dos analitos, os testes foram realizados utilizando-se a temperatura de $25^{\circ} \mathrm{C}$ com biomassas variando de 0,03 a $0,50 \mathrm{~g}$.

Os resultados obtidos são apresentados na Figura 2, onde se observa que a retenção do TBT pela levedura ocorreu em quantidades superiores a $90 \%$ para todas as quantidades de biomassa testadas e que a presença do composto na fase líquida continuou apresentando valores inferiores ao LD. A retenção do Sn(IV) pela levedura foi ínfima, apresentando variações pouco significativas entre as diferentes condições de extração, principalmente para pequenos valores de biomassa. Assim, considerando-se os resultados, foi adotada a massa de $0,0625 \mathrm{~g}$ de levedura para os próximos experimentos.

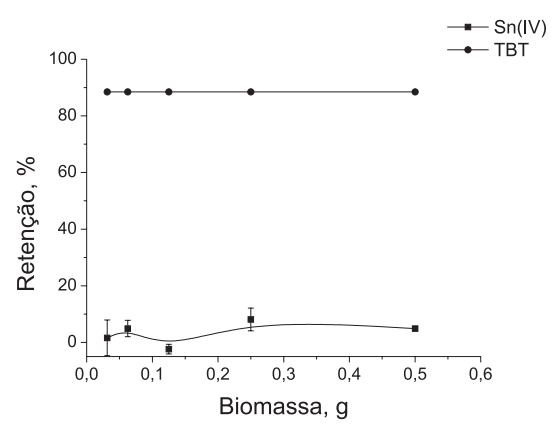

Figura 2. Efeito da biomassa na retenção de TBT e Sn(IV). Temperatura = $25^{\circ} \mathrm{C}$; tempo de contato $=0 \mathrm{~min} ; \mathrm{pH}=6 \pm 0,5$

\section{Efeito da temperatura}

Os experimentos para a avaliação do efeito da temperatura foram conduzidos segundo os parâmetros pré-estabelecidos (biomassa de $0,0625 \mathrm{~g}$ e extração imediata). As soluções teste de $\mathrm{Sn}(\mathrm{IV})$ ou TBT foram colocadas em banho (de água) com temperatura controlada variando entre 20 e $50{ }^{\circ} \mathrm{C}$; no momento em que atingiram as temperaturas desejadas, foram transferidas para os tubos contendo a levedura.

As retenções dos analitos pela levedura em função da temperatura de incubação são apresentadas na Figura 3. Observa-se um discreto aumento na retenção de $\mathrm{Sn}$ (IV) com o aumento da temperatura, porém, em todos os casos, a retenção da espécie foi menor que $7,8 \%$. No caso do TBT, a presença do composto na fase líquida só foi observada em temperaturas de 30 e $50{ }^{\circ} \mathrm{C}$.

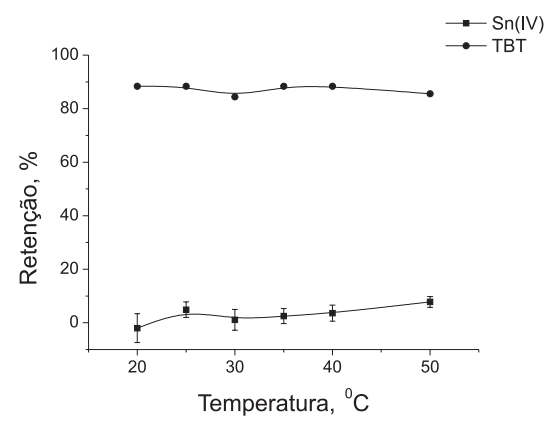

Figura 3. Efeito da temperatura na retenção de TBT e Sn(IV). Biomassa = $0,0625 \mathrm{~g}$; tempo de contato $=0 \mathrm{~min} ; \mathrm{pH}=6 \pm 0,5$

\section{Geração de $\mathrm{SnH}_{4}$ após extração em fase sólida usando $S$.} cerevisiae

As condições de operação do sistema de geração de hidretos foram definidas a partir de trabalhos anteriores. ${ }^{45-47}$ Presumiu-se que uma concentração de $\mathrm{NaBH}_{4}$ a $3 \%\left(0,8 \mathrm{~mol} \mathrm{~L}^{-1}\right)+\mathrm{NaOH} 0,05 \mathrm{~mol} \mathrm{~L}^{-1}$ seria suficiente para resultar em uma relação molar recomendada. ${ }^{45}$ Também, visando minimizar os valores do branco evitou-se trabalhar com concentrações de $\mathrm{NaBH}_{4}$ superiores a $3 \%(\mathrm{~m} / \mathrm{v})$.

Visando minimizar os efeitos da matéria orgânica presente na fase sólida, foi realizada uma digestão parcial das amostras com $\mathrm{HNO}_{3}$ (pré-tratamento descrito anteriormente) para que a determinação de TBT fosse satisfatória.

Na Tabela 2 são apresentados os parâmetros de mérito para a determinação de TBT por ICP OES, usando nebulização pneumática e geração de hidretos.

Tabela 2. Parâmetros de mérito para determinação de TBT por ICP OES

\begin{tabular}{lcc}
\hline & $\begin{array}{c}\text { Nebulização } \\
\text { Pneumática }\end{array}$ & $\begin{array}{c}\text { Geração } \\
\text { de Hidretos }\end{array}$ \\
\hline LOD, $\mu \mathrm{g} \mathrm{L}{ }^{-1}$ & 16,3 & 1,9 \\
$\mathrm{LOQ}, \mu \mathrm{g} \mathrm{L}{ }^{-1}$ & 53,3 & 6,3 \\
Sensibilidade, contagens $/ \mu \mathrm{g} \mathrm{L}{ }^{-1}$ & 5,3 & 119,5 \\
\hline
\end{tabular}

Na Figura 4 é apresentada a curva analítica para TBT. A curva foi obtida com soluções contendo entre 0 e $25 \mu \mathrm{g} \mathrm{L}^{-1}$ do analito submetido aos processos de extração em fase sólida e digestão parcial com $\mathrm{HNO}_{3}$. Para a faixa de trabalho $(2,5-25 \mu \mathrm{g}$ $\mathrm{L}^{-1}$ ) observa-se uma correlação linear caracterizada por um $\mathrm{R}^{2} \mathrm{de}$ 0,98 . Com a geração de hidretos, a sensibilidade para Sn $(119 \mu \mathrm{g}$ $\mathrm{L}^{-1}$ ) aumentou consideravelmente em comparação à nebulização pneumática (superior a 20 vezes). Foi obtido um limite de detecção (3 $\sigma)$ de $1,90 \mu \mathrm{g} \mathrm{L}^{-1}$.

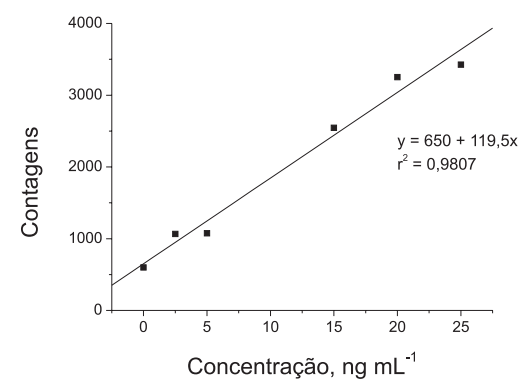

Figura 4. Curva analítica para TBT obtida com a geração de hidretos

\section{Interferências}

$\mathrm{O}$ teste de interferência foi conduzido submetendo-se soluções contendo $25 \mu \mathrm{g} \mathrm{L}^{-1}$ de TBT e o potencial interferente ao processo de extração em fase sólida e à geração de hidretos. Foram avaliados os efeitos interferentes dos seguintes elementos: $\mathrm{Cu}$ (II), $\mathrm{Fe}(\mathrm{III})$, $\mathrm{Cd}(\mathrm{II}), \mathrm{Mn}(\mathrm{II}), \mathrm{Zn}(\mathrm{II}), \mathrm{Ca}(\mathrm{II}), \mathrm{Mg}(\mathrm{II}), \mathrm{K}(\mathrm{I})$ e $\mathrm{Na}(\mathrm{I})$. As recuperações (Tabela 3) foram calculadas tendo como base os sinais provenientes de medidas na fase sólida de uma solução contendo o analito sem os potenciais interferentes.

Os efeitos interferentes negativos (supressão de sinal) devido à presença de metais de transição são típicos de processo de geração de hidretos. ${ }^{45}$ Deste modo pode-se inferir que o processo interferente de $\mathrm{Cu}, \mathrm{Fe}, \mathrm{Cd}, \mathrm{Mn}$ e $\mathrm{Zn}$ na determinação de TBT foi minimizado devido à extração em fase sólida. Por outro lado, a presença de metais alcalinos e alcalinos terrosos, particularmente $\mathrm{Mg}$, resulta em uma tendência de interferência positiva na determinação de TBT (Tabela 3). 
Tabela 3. Interferência de íons contaminantes na recuperação de TBT na fase sólida $\left(25 \mu \mathrm{g} \mathrm{L}^{-1}\right)$

\begin{tabular}{lccc}
\hline Composto & Interferente & $\begin{array}{c}\text { Razão, } \\
\text { Interferente:Sn }\end{array}$ & Recuperação, \% \\
\hline $\mathrm{CuSO}_{4} \cdot 5 \mathrm{H}_{2} \mathrm{O}$ & $\mathrm{Cu}(\mathrm{II})$ & 80 & $112 \pm 22$ \\
$\mathrm{FeNH}_{4}\left(\mathrm{SO}_{4}\right)_{2} \cdot 12 \mathrm{H}_{2} \mathrm{O}$ & $\mathrm{Fe}(\mathrm{III})$ & 80 & $118 \pm 5$ \\
$\mathrm{Cd}_{\left(\mathrm{NO}_{3}\right)_{2}}$ & $\mathrm{Cd}(\mathrm{II})$ & 80 & $105 \pm 8$ \\
$\mathrm{MnSO}_{4} \cdot \mathrm{H}_{2} \mathrm{O}$ & $\mathrm{Mn}(\mathrm{II})$ & 80 & $108 \pm 3$ \\
$\mathrm{ZnSO}_{4} \cdot 7 \mathrm{H}_{2} \mathrm{O}$ & $\mathrm{Zn}(\mathrm{II})$ & 80 & $126 \pm 10$ \\
$\mathrm{CaCO}_{3}$ & $\mathrm{Ca}(\mathrm{II})$ & 200 & $104 \pm 10$ \\
$\mathrm{MgCl}_{2} \cdot \mathrm{H}_{2} \mathrm{O}$ & $\mathrm{Mg}(\mathrm{II})$ & 200 & $150 \pm 24$ \\
$\mathrm{KCl}_{\mathrm{NaCl}}$ & $\mathrm{K}(\mathrm{I})$ & 800 & $121 \pm 21$ \\
& $\mathrm{Na}(\mathrm{I})$ & 800 & $122 \pm 14$ \\
\hline
\end{tabular}

\section{Determinação de TBT na presença de Sn(IV) em amostras de} águas

Para avaliar o desempenho do método desenvolvido para determinação de TBT na presença de Sn(IV) em amostras de água, não sendo disponíveis amostras certificadas, foram realizados testes de recuperação onde as amostras foram enriquecidas com TBT e $\mathrm{Sn}(\mathrm{IV})$. Foram analisadas 3 amostras: uma amostra sintética, uma de água de rio e uma de água do mar. A amostra sintética foi preparada adicionando-se $10 \mu \mathrm{g} \mathrm{L}^{-1}$ dos analitos em água desionizada $(18,2$ $\mathrm{M} \Omega \mathrm{cm})$. Também, para as amostras de água de rio e do mar foram adicionados $10 \mu \mathrm{g} \mathrm{L}^{-1}$ do analito antes dos procedimentos de extração e pré-tratamento. As concentrações de TBT foram quantificadas a partir de curva analítica construída com soluções contendo de 0 a $25 \mu \mathrm{g}$ $\mathrm{L}^{-1}$. Visando excluir qualquer interferência na determinação causada pelo processamento das amostras, essas soluções foram submetidas às etapas de extração em fase sólida e pré-tratamento com ácido nítrico juntamente com as amostras.

Na Tabela 4 são apresentadas as recuperações obtidas na determinação de TBT nas amostras de água analisadas. Verifica-se que para todas as amostras as recuperações de TBT foram satisfatórias.

Tabela 4. Determinação de TBT em amostras de água e material biológico

\begin{tabular}{lccc}
\hline Amostra & $\begin{array}{c}\text { Massa } \\
\text { adicionada, ng }\end{array}$ & $\begin{array}{c}\text { Massa } \\
\text { encontrada, ng }\end{array}$ & $\begin{array}{c}\text { Recuperação, } \\
\%\end{array}$ \\
\hline Sintética & 10 & $12,9 \pm 1,0$ & $129 \pm 8$ \\
Água do rio & 10 & $11,4 \pm 0,6$ & $114 \pm 6$ \\
Água do mar & 10 & $10,1 \pm 1,0$ & $101 \pm 10$ \\
Brânquias de siri & 500 & $432 \pm 45$ & $86 \pm 9$ \\
\hline
\end{tabular}

Considerando que o $\mathrm{Sn}(\mathrm{IV})$ pode ser seletivamente determinado na fase líquida, também foram realizados testes para determinar o analito nas amostras de águas sintética, de rio e de mar. Os resultados apresentaram boas recuperações para água sintética e de rio ( 92 e $84 \pm 17 \%$, respectivamente), porém, para a água do mar a determinação do Sn inorgânico se mostrou insatisfatória $(33 \pm 6 \%)$. Deste modo, uma possível abordagem para o fracionamento químico de Sn inorgânico e orgânico (TBT) só pode ser aplicada para análise de amostras de água de rio.

\section{Determinação de TBT na presença de $\operatorname{Sn}(I V)$ em brânquias de siri}

Os testes de recuperação do analito foram conduzidos com a adição de 500 ng de TBT (e de $\operatorname{Sn}(\mathrm{IV})$ ) às brânquias maceradas de siri azul que ficaram em contato por $45 \mathrm{~min}$. Então, o tecido macerado foi submetido ao procedimento para extração do analito.

Do extrato enriquecido, alíquotas de $10 \mathrm{~mL}$ foram adicionadas aos tubos contendo levedura, para o processo de extração em fase sólida e, em seguida, ao pré-tratamento para determinação por geração de hidretos. Neste caso, os analitos foram quantificados com soluções contendo TBT com concentrações variando de 0 a $100 \mu \mathrm{g} \mathrm{L}^{-1}$, que passaram pelo mesmo processo das amostras (extração em fase sólida e pré-tratamento com ácido nítrico).

As recuperações de TBT em brânquias de siri são apresentadas na Tabela 4. Para a determinação de TBT o procedimento desenvolvido mostrou-se eficiente, apresentando recuperação satisfatória. Similarmente ao ocorrido para as amostras de água de mar, a recuperação de $\mathrm{Sn}(\mathrm{IV})$ no material biológico não foi satisfatória $(16 \pm 2 \%)$.

\section{CONCLUSÕES}

Os resultados obtidos mostraram que a levedura Saccharomyces cerevisiae pode ser utilizada para separação de TBT e Sn(IV) em soluções padrão usando extrações em fase sólida em batelada, pois, em determinadas condições, o primeiro composto é retido pela levedura, enquanto o segundo permanece em solução.

Foi desenvolvido um novo método para determinação seletiva de TBT na presença de $\mathrm{Sn}$ (IV) em amostras de águas fluviais, marinhas e material biológico. O método é relevante para análises de amostras de interesse ambiental, principalmente, se considerado que o TBT é uma das espécies mais tóxicas de $\mathrm{Sn}$.

\section{AGRADECIMENTOS}

À Fundação de Amparo à Pesquisa do Estado de São Paulo (FAPESP) pelo apoio financeiro.

\section{REFERÊNCIAS}

1. Leroy, M. J. F.; Quevauviller, P.; Donard, O. F. X.; Astruc, M.; Pure Appl. Chem. 1998, 70, 2051.

2. Harison, R. M.; Rapsomanikis, S.; Environmental Analysis using Chromatography Interfaced with Atomic Spectroscopy, Ellis Howood: Chischester, 1989.

3. Shpigun, L. K.; Lunina, V. K.; J. Anal. Chem. 2003, 58, 1078.

4. Huggett, R. J.; Unger, M. A.; Seligman, P. F.; Valkirs, A. O.; Environ. Sol. Technol. 1992, 26, 232.

5. Rajendran, R. B.; Tao, H.; Miyazaki, A.; Ramesh, R.; Ramachandran, S.; J. Environ. Monit. 2001, 3, 627.

6. Wilken, R. D.; Kubala, J.; Jantzen, E.; J. Anal. Chem. 1994, 350, 77.

7. Jiang, G. B.; Zhou, Q. F.; J. Chromatogr., A 2000, 886, 197.

8. Mester, Z.; Sturgeon, R.; Pawliszyn, J.; Spectrochim. Acta, Part B 2001, 56, 233.

9. Pereiro, I. R.; Díaz, A. C.; Anal. Bioanal. Chem. 2002, 372, 74.

10. Vinas, P.; Lopez-Garcia, I.; Merino-Merono, B.; Campillo, N.; Hernandez-Cordoba, M.; J. Environ. Monit. 2004, 6, 262.

11. Ichinoki, S.; Iwase, H.; Arakawa, F.; Hirano, K.; Fuji, Y.; J. Liq. Chromatogr. Relat. Technol. 2003, 26, 3129.

12. Grotti, M.; Rivaro, P.; Frache, R.; J. Anal. At. Spectrom. 2001, 16, 270.

13. Rivas, C.; Ebdon, L.; Evans, E. H.; Hill, S. J.; Appl. Organomet. Chem. 1996, 10, 61 .

14. Yang, H. J.; Jiang, S. J.; Yang, Y. J.; Hwang, C. J.; Anal. Chim. Acta 1995, 312, 141.

15. Caruso, J. A.; Montes-Bayon, M.; Ecotoxicol. Environ. Saf. 2003, 56, 148.

16. Bermejo-Barrera, P.; Anllo-Sendin, R. M.; Cantelar-Barbazan, M. J.; Bermejo- Barrera, A.; Anal. Bioanal. Chem. 2002, 372, 837.

17. Bermejo-Barrera, P.; Gonzalez-Campos, G.; Ferron-Novais, M.; Bermejo-Barrera, A.; Talanta 1998, 46, 1479.

18. Vandenberg, C. M. G.; Khan, S. H.; Analyst 1991, 16, 585.

19. Han, J. S.; Weber, J. H.; Anal. Chem. 1998, 60, 316. 
20. Takahashi, Y.; Sakakibara, N.; Nomura, M.; Anal. Chem. 2004, 76, 4307.

21. He, B.; Wu, D.; Jiang, G. B.; Spectrosc. Spect. Anal. 1999, 19, 718.

22. Ni, Z. M.; Hang, H. B.; Li, A.; He, B.; Xu, F. Z.; J. Anal. At. Spectrom. 1991, 6, 385.

23. Imura, H.; Suzuki, N.; Anal Chem. 1983, 55, 1107.

24. Madrid, Y.; Cabrera, C.; Pérez-Corona, T.; Cámara, C.; Anal. Chem. 1995, 67, 750 .

25. Pérez-Corona, T.; Madrid, Y.; Cámara, C.; Anal. Chim. Acta 1997, 345, 249.

26. Smichowski, P.; Marrero, J.; Ledesma, A.; Polla, G.; Batistone, D. A.; J. Anal. At. Spectrom. 2000, 15, 1493.

27. Bag, H.; Lale, M.; Turker, R.; Tunceli, A.; Talanta 2000, 51, 895.

28. Menegário, A. A.; Smichowski, P.; Polla, G.; Anal. Chim. Acta 2005, 546, 244.

29. Péres-Corona, T.; Madrid-Albarrán, Y.; Cámara, C.; Beceiro, E.; Spectrochim. Acta, Part B 1998, 53, 321 .

30. Koh, J.; Kwon, Y.; Pak, Y.; Microchem. J. 2005, 80, 195.

31. Menegário, A. A.; Tonello, P. S.; Biscaro, P. A.; Brossi-Garcia, A. L.; Microchim. Acta 2007, 159,247.

32. Caldorin, R.; Menegário, A. A.; Microchim. Acta 2007, 157, 201.

33. Cabral, L. M.; Juliano, V. N. M.; Dias, L. R. S.; Dornelas, C. B.; Rodrigues, C. R.; Villardi, M.; Castro H. C.; dos Santos, T.C.; Mem. I. Oswaldo Cruz 2008, 103, 130.

34. Menegário A. A.; Smichowski, P.; Tonello, P. S.; Polla, G.; Oliveira, E. P.; Santelli, R. E.; Anal. Chim. Acta 2008, 625, 131.
35. dos Santos, E. J.; Herrmann A. B.; de Caires, S. K.; Frescura V. L. A.; Curtius, A. J.; Spectrochim. Acta, Part B 2009, 64, 549.

36. Lopes, W. D.; Santelli, R. E.; Oliveira E. P.; Carvalho M. D. P.; Bezerra M. A.; Talanta 2009, 79, 1276.

37. Benzo, Z.; Maldonado, D.; Chirinos J.; Marcano E.; Gómez C.; Quintal, M.; Salas J.; Microchem. J. 2009, 93, 127.

38. Tyburska, A.; Jankowski, K.; Ramsza, A.; Reszke, E.; Strzelec, M.; Andrzejczuk A; J. Anal. At. Spectrom. 2010, 25, 210.

39. Antolin, R.; Borge, G.; Posada, T.; Etxebarria, N.; Raposo, J. C.; J. Alloys Compd. 2007, 427, 73.

40. Pohl, P.; Lesniewicz, A.; Zyrnicki, W. , Int. J. Environ. Anal. Chem. 2003, 83, 963 .

41. Hosick, T. J.; Ingamells, R. L.; Machemer, S. D.; Anal. Chim. Acta 2002, 456, 263.

42. Menegário, A. A.; Giné, M. F.; Spectrochim. Acta, Part B 2000, 55, 355.

43. Quevauviller, Ph.; Morabito, R.; Trends Anal. Chem. 2000, 19, 86.

44. Caldorin, R.; Menegário, A. A.; 2005 European Winter Conference on Plasma Spectrometry, Budapeste, Hungria, 2005.

45. Dedina, J.; Tsalev, D.; Hydride Generation Atomic Absorption Spectrometry, John Wiley \& Sons: England, 1995, vol. 130, p. 526.

46. Mandjukov, P. B.; Djarkova, V.; Tsalev, D. L.; 6. Colloquium Atomspektrometrische Spurenanalytik,Ueberlinger, Alemanha, 1991.

47. Qiu, D. R.; Vandecasteele, C.; Vermeiren, K.; Dams, R.; Spectrochim. Acta, Part B 1990, 45, 439. 